\title{
A Comparison of Different Estimation Methods for Hysteresis Modelling
}

\author{
R. JAstrzęBSki ${ }^{a}$, K. ChWASteK $^{a}$, I. Biondić ${ }^{b}$ And K. MiliČeviĆ ${ }^{b}$ \\ ${ }^{a}$ Faculty of Electrical Engineering, Częstochowa University of Technology, \\ al. Armii Krajowej 17, 42-201 Częstochowa, Poland \\ ${ }^{b}$ Faculty of Electrical Engineering, J.J. Strossmayer University of Osijek, 31000 Osijek, Croatia
}

\begin{abstract}
The paper focuses on the estimation techniques for a low-dimensional phenomenological model of ferromagnetic hysteresis proposed by the Brazilian research group GRUCAD. The description is expressed with an ordinary differential equation and some auxiliary relationships. It describes both irreversible and reversible magnetization processes and addresses some problems inherent in the well-known Jiles-Atherton model. It is found that the differential evolution method is the most competitive technique for recovery of optimal model parameters.
\end{abstract}

DOI: 10.12693/APhysPolA.131.1228

PACS/topics: 75.60.-d, 75.60.Ej

\section{Introduction}

An important stage of hysteresis modeling is the identification of model parameters. For this purpose, different optimization techniques, including those based on artificial intelligence methods and soft computing, are applied [1-3]. An improper choice of values for model parameters may result in excessive prediction errors if other phenomena (e.g. eddy currents) are taken into account [4]. On the other hand, different sets of model parameters may yield identified hysteresis loops that are quite similar in shape [5]. The present paper is aimed at the development of a methodology to choose an optimum procedure to estimate the parameters of a low dimensional hysteresis model, described in Refs. [6-8].

\section{Model description}

The description advanced by Jiles and Atherton [9] has attracted a lot attention of the scientific community in the last thirty years. In the present paper, we focus on its modification proposed by the Brazilian team GRUCAD [6, 7], which has addressed a number of problems with the original description. As pointed out in Ref. [10], in the original Jiles-Atherton (JA) model the loop branches are obtained by introducing offsets along the $M$-axis. On the contrary, the GRUCAD proposal is based on introducing offsets along the $H$-axis from the so-called anhysteretic (truly reversible) curve. Therefore the latter model yields an improved description of reversible magnetization processes in the sense of thermodynamics, for example there is no need to introduce artificial "switching off" of the irreversible magnetization component after sudden field reversals. The slope of the magnetization curve described with the GRUCAD model is non-zero after a field reversal, such model behavior is expected for the advanced Preisach-based models like the moving Preisach model [11].

Yet another practical advantage of the GRUCAD description is its formulation directly as $B$-input model, which is in compliance with international standards concerning magnetic measurements.

The model equations are:

(1) $H_{a n}=B / \mu_{0}-M_{s}(\operatorname{coth} \lambda-1 / \lambda)$,

(2) $\lambda=\frac{(1-\alpha) H_{a n}+\alpha B / \mu_{0}}{a}$,

(3) $\frac{\mathrm{d} H_{h}}{\mathrm{~d} B}=\frac{H_{H S}\left(\operatorname{coth} \lambda_{H}-1 / \lambda_{H}\right)+H_{h}}{\gamma \delta}$, where

$\delta= \pm 1=\operatorname{sgn} \frac{\mathrm{d} H_{h}}{\mathrm{~d} B}$,

(4) $\lambda_{H}=\frac{H_{h}+\delta H_{H S}}{a}$,

(5) $H=H_{a n} \stackrel{a}{+} H_{h}$.

The model parameters are $\alpha, a, \gamma, H_{H S}$ and $M_{s}$. The paper [8] provides information how the variation of the above-mentioned parameters qualitatively affects the shape of the modeled hysteresis loop.

\section{Comparison of different estimation techniques}

In order to compare the efficiency of different estimation techniques, we propose the following procedure:

1. estimation of model parameters using a generated loop with a priori preset parameters,

2. the same as in previous point, but an artificially generated noise is added to the values for the reference point to simulate inevitable measurement errors,

3. estimation of model parameters for a real life sample.

For comparison we choose the following methods: differential evolution, genetic algorithms, particle swarm optimization and simulated annealing. All algorithms have been implemented as components of the Matlab toolbox GODLIKE (Global Optimum Determination by Linking and Interchanging Kindred Evaluators) by Oldenhuis [12].

Both genetic algorithms and differential evolution are stochastic global optimization methods inspired by the 
evolution processes observed in nature. Genetic algorithm (GA) transforms a population or a set of individual objects, each with an associated fitness value, into a new generation of the population using the Darwinian principles of reproduction and survival of the fittest and analogs of naturally occurring genetic operations such as crossover (sexual recombination) and mutation. Each individual in the population represents a possible solution to a given problem. The genetic algorithm attempts to find an optimal solution to the problem in a number of iterations corresponding to successive generations. In every generation the most promising (fittest) individuals are chosen to produce offsprings $[13,14]$. The block diagram illustrating the general flow chart in GA is shown in Fig. 1.

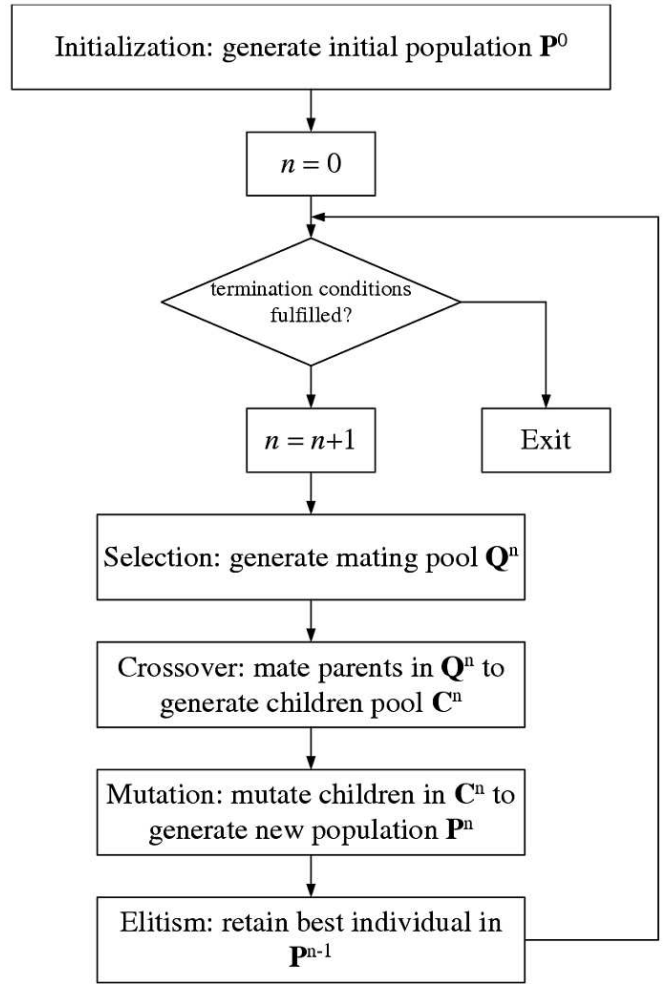

Fig. 1. The general flow chart of genetic algorithms (own work, after [14]).

Nowadays GA is considered a bit of an outdated technique (originally proposed by Holland in 1975), in most cases it has been successfully superseded by the more recent and effective differential evolution. Genetic algorithms, either in the binary or floating number representation, have been successfully applied for the task of recovery of optimal values of JA model parameters in the papers [1, 15-19].

Differential evolution (DE) was proposed in the midnineties of the last century by Price as a way to address some shortcomings of the GAs (premature convergence, slow performance). The key difference between DE and $\mathrm{GA}$ is the introduction of the differential mutation op- erator, whose role is to maintain the self-adaptability of trial solutions [20-22]. DE is now considered one of the most robust and versatile metaheuristic optimization techniques.

The pseudo-code of DE is given below [22].

Require: $D$ - problem dimension (optional)

$N P, F, C r$ - control parameters

$G E N$ - stopping condition

$L, H$ - boundary constraints

Initialize population $P O P_{i, j} \leftarrow \operatorname{rand}_{i, j}[L, H]$ and

Evaluate fitness $F I T_{j} \leftarrow f\left(P O P_{j}\right)$

for $g=1$ to $G E N$ do

for $j=1$ to $N P$ do

Choose randomly $r_{1,2,3} \in[1, \ldots, N P], r_{1} \neq r_{2} \neq r_{3} \neq j$

Create trial individual $X \leftarrow S(r, F, C r, P O P)$

Verify boundary conditions if $\left(x_{i} \notin[L, H]\right)$

$x_{i} \leftarrow \operatorname{rand}_{i}[L, H]$

Select better solution $\left(X\right.$ or $\left.P O P_{j}\right)$, and update $i B e s t$ if required

end for

end for

Several authors report on successful application of differential evolution to the problem of estimation of JA model parameters [23-25].

Particle swarm optimization (PSO) is a populationbased stochastic optimization technique, inspired by social behavior of bird flocks or fish schools. Since 1995, when the original concept was proposed [26], a number of important improvements have been introduced [14, 27]. The basic concept of this method is to randomly generate a number of potential solutions (called particles or agents) of the optimization problem in the $n$-dimensional search space and next to seek for an improved one by moving them simultaneously in accordance with simple mathematical formulae. Particles with best features move slower and attract the other ones. After a number of iterations the optimal solution, indicated by swarming candidates, is determined. PSO has been applied for estimation of JA model parameters by Marion et al. [28] and Knypiński et al. [29].

Simulated annealing (SA) is a random search technique that mimics the annealing process in metallurgy [30, 31]. In statistical mechanics the behaviour of large aggregates of atoms subject to cooling is highly influenced by the cooling rate. A slow decrease of sample temperature results in the smearing out of the structural disorder and favours the approach to an equilibrium state corresponding to the global energy minimum. On the other hand, rapid cooling (quenching) yields defects and glass-like intrusions inside the material. It leads to the final state with a higher energy level than previously considered. The main advantage of simulated annealing is its ability to avoid being trapped in local minima. Under proper control of the cooling rate the SA method shall converge to the global minimum. This technique has been applied for estimation of JA model parameters by several authors [32-34]. 


\section{Results}

For a virtual dataset of model parameters $\left[\alpha, a, M_{S}, \gamma, H_{H S}\right]=\left[10^{-6}, \quad 50, \quad 1.1 \times 10^{6}, \quad 0.1\right.$, 100] all algorithms implemented in the GODLIKE toolbox were run 20 times. The repeatability of each algorithm was verified by a direct comparison of the collected results. As optimum results the average values of model parameters were assumed. The fitness function was defined as the sum of squared errors between the measured and the modeled values for 33 data points belonging to the descending branch of the major hysteresis loop. Default ("out-of-the-box") settings for all algorithms were used. In terms of accuracy, the most competitive algorithm was DE (15 times the winner and the best fitness at all), the worst was SA. GA was winner twice, but for 17 times the method produced second-to-one results. PSO was the winner three times. It is remarkable that DE yielded the most robust (repetitive) results. In terms of computational cost PSO required the least number of iterations and function calls, GA was slightly behind. SA availed of the resources most. The practical indication for possible users is that DE seems the best method for the purpose of GRUCAD model identification.

In the next step, artificial $10 \%$ noise was added to the reference hysteresis loop and the algorithms were re-run again. For the distorted hysteresis loop, the advantages of DE were not so obvious in comparison to PSO and GAs. The three methods yielded quite comparable final values of fitness and the computational burden measured with the number of iterations and function calls was quite similar. SA was the weakest competitor again. It seemed to scan quite different areas of the search space. We suspect the improvement of its performance might be possible by tweaking the cooling schedule to be applied at each iteration. According to the documentation [12], the simulated annealing routine implemented in the GODLIKE toolbox is probably the weakest algorithm for single-objective optimization, as the adaptation of control parameters at each iteration is not implemented yet. Our results confirm the statement of toolbox developer Oldenhuis.

The considered optimization techniques were next applied to the estimation of the GRUCAD model parameters for a cylinder sample made of $98 \mathrm{NiFe}$ (permalloy). The parameters were estimated using the major hysteresis loop measured at quasi-static conditions, $B_{m}=0.6 \mathrm{~T}$. The bounds for model parameters were determined using the empirical guidelines as given in Ref. [2]. The bounds for the parameter $\gamma$ were set to $[0.01 ; 0.5]$, whereas for the parameter $H_{H S}-[2 ; 7]$ (its value should correspond to the measured quasi-static coercivity). Figure 2 depicts the best solution obtained during the tests.

The estimated set of model parameters was obtained, as previously, with the use of DE. The estimated model parameters were $\alpha=3.67 \times 10^{-5}, a=7.28 \mathrm{~A} / \mathrm{m}, M_{s}=$ $5.799 \times 10^{5} \mathrm{~A} / \mathrm{m}, \gamma=0.039 \mathrm{~T}$ and $H_{H S}=2.64 \mathrm{~A} / \mathrm{m}$. The

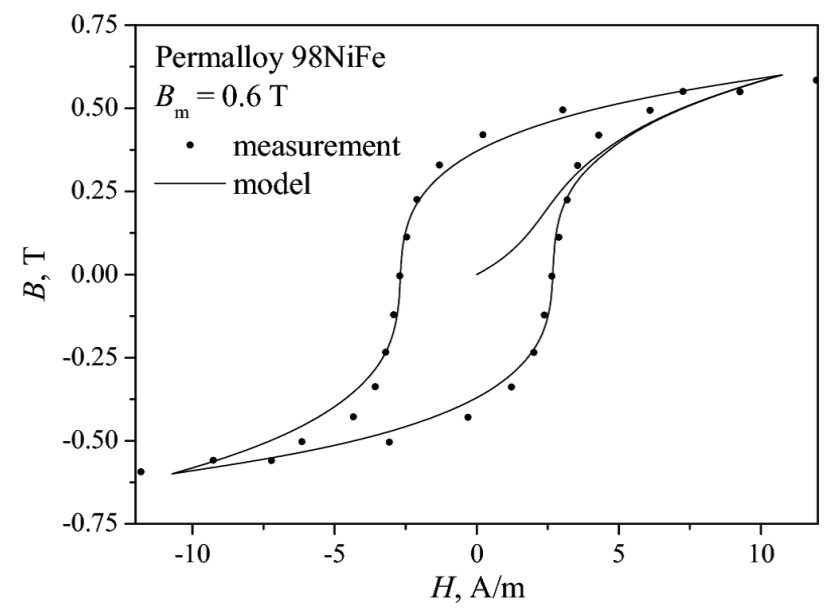

Fig. 2. The measured and the modeled hysteresis loop for $98 \mathrm{NiFe}$ sample.

TABLE I

Percentage errors in chosen characteristic points

\begin{tabular}{c|c|c|c|c}
\hline \hline Point on the loop & $B_{r}$ & $H_{c}$ & $0.5 H_{\max }$ & $-0.5 H_{\max }$ \\
\hline Error [\%] & 9.2 & 0.4 & 0.3 & 5.6
\end{tabular}

values of percentage errors in chosen points belonging to the upper branch of hysteresis loop are given in Table I.

As far as the performance of the algorithms is concerned, the timing for the whole batch varied during the tests from 40 to $195 \mathrm{~s}$ on an Intel i5-2430M, 4GB machine running Matlab R2013a on Windows 7 platform. DE was the winner, but GA and PSO were right behind on the podium (GA was slightly more efficient).

\section{Conclusions}

1. Four metaheuristic algorithms implemented by the same developer were compared in terms of their ability to recover the optimal values of model parameters.

2. A methodology for comparison of stochastic estimation methods used for estimation of the GRUCAD model parameters was proposed.

3. The differential evolution method seems to be the most competitive algorithm for the purpose.

\section{References}

[1] F. Riganti Fulginei, A. Salvini, IEEE Trans. Magn. 41, 1100 (2005).

[2] K. Chwastek, J. Szczygłowski, Przegl. Elektrotechn. 12, 145 (2008).

[3] R.-A. Naghizadeh, B. Vahidi, S.H. Hosseinian, COMPEL 31, 1293 (2012). 
[4] S. Steentjes, M. Petrun, D. Dolinar, K. Hameyer, IEEE Trans. Magn. 52, 7300804 (2016).

[5] G.M. Lozito, A. Salvini, in: AEIT Annual Conf. From Research to Industry: The Need for a More Effective Technology Transfer (AEIT), Trieste (Italy), 2014, p. 1.

[6] P.I. Koltermann, L.A. Righi, J.P.A. Bastos, R. Carlson, N. Sadowski, N.J. Batistela, Physica B 275, 233 (2000).

[7] L.A. Righi, N. Sadowski, R. Carlson, J.P.A. Bastos, N.J. Batistela, IEEE Trans. Magn. 37, 3353 (2001).

[8] S. Steentjes, K. Chwastek, M. Petrun, D. Dolinar, K. Hameyer, IEEE Trans. Magn. 50, 7300804 (2014).

[9] D.C. Jiles, D.L. Atherton, J. Magn. Magn. Mater. 61, 48 (1986)

[10] S.E. Zirka, Yu.I. Moroz, R.G. Harrison, K. Chwastek, J. Appl. Phys. 112, 043916 (2012).

[11] E. Della Torre, Magnetic Hysteresis, IEEE Press, Piscataway 2000 .

[12] R. Oldenhuis, GODLIKE Matlab toolbox.

[13] D. Goldberg, Genetic Algorithms in Search, Optimization, and Machine Learning, Addison-Wesley, Reading 1989.

[14] Xin-She Yang, Engineering Optimization. An Introduction with Metaheuristic Applications, Wiley, Hoboken (NJ) 2010.

[15] P.R. Wilson, J. Neil Ross, A.D. Brown, IEEE Trans. Magn. 37, 989 (2001).

[16] J.V. Leite, S.L. Avila, N.J. Batistela, W.P. Carpes, N. Sadowski, P. Kuo-Peng, J.P.A. Bastos, IEEE Trans. Magn. 40, 888 (2004).

[17] K. Chwastek, J. Szczygłowski, Math. Comp. Simulat. 71, 206 (2006).

[18] A.P.S. Baghel, S.V. Kulkarni, IET Electr. Power Appl. 6, 689 (2012).

[19] Lu Hai-Liang, Wen Xi-Shan, Lan Lei, An Yun-Zhu, Li Xiao-Ping, J. Magn. Magn. Mater. 374, 502 (2015).
[20] K.V. Price, R.M. Storm, J.A. Lampinen, Differential Evolution. A Practical Approach to Global Optimization, Springer-Verlag, Berlin 2005.

[21] Anyong Qing, Differential Evolution: Fundamentals and Applications in Electrical Engineering, Wiley, Singapore 2009.

[22] V. Feoktistov, Differential Evolution. In Search of Solutions, Springer, New York 2006.

[23] M. Toman, G. Štumberger, D. Dolinar, IEEE Trans. Magn. 44, 1098 (2008).

[24] L. dos Santos Coelho, V. Cocco Mariani, J.V. Leite, Expert Syst. Appl. 39, 2021 (2012).

[25] R. Biedrzycki, R. Szewczyk, P. Švec Sr., W. Winiarski, chapter in: Mechatronics Ideas for Industrial Application, series Advances in Intelligent Systems and Computing, Vol. 317, Springer-Verlag, 2015, p. 11.

[26] J. Kennedy, R. Eberhart, in: Proc. IEEE Int. Conf on Neural Networks, Perth (Australia), 1995, Vol. IV, p. 1942.

[27] Particle Swarm Optimization, Ed. A. Lazinica, InTech, Rijeka 2009.

[28] R. Marion, R. Scorretti, N. Siauve, M.-A. Raulet, L. Krähenbühl, IEEE Trans. Magn. 44, 894 (2008).

[29] Ł. Knypiński, L. Nowak, P. Sujka, K. Radziuk, Arch. Electr. Eng. 61, 139 (2012).

[30] S. Kirkpatrick, C.D. Gelatt Jr., M.P. Vecchi, Science 220, 671 (1983).

[31] A. Corana, M. Marchesi, C. Martini, S. Ridella, ACM Trans. Math. Software 13, 262 (1987).

[32] D. Lederer, H. Igarashi, A. Kost, T. Honma, IEEE Trans. Magn. 35, 1211 (1999).

[33] E. Del Moral Hernandez, C.S. Muranaka, J.R. Cardoso, Physica B 275, 212 (2000).

[34] Baodong Bai, Jiayin Wang, Keqing Zhu, in: Proc. 2011 Int. Conf. on Electr. Machines and Syst., ICEMS'2011. 